\title{
Hepatic phosphoenolpyruvate carboxykinase expression after gastric bypass surgery in rats with type 2 diabetes mellitus
}

\author{
J. Wu' ${ }^{1}$, S.S. Hou ${ }^{1 *}$, W. Wang ${ }^{2 *}$, M. Yin ${ }^{2}$, N. Cheng ${ }^{2}$, L.L. $\mathrm{Ge}^{2}$, J.J. Yin ${ }^{2}$ and J. $\mathrm{Xu}^{2}$ \\ 'Zunyi Medical University, Zunyi, China \\ ${ }^{2}$ Zhongshan Hospital Affiliated of Dalian University, Dalian, China \\ *These authors contributed equally to this study. \\ Corresponding authors: J.J. Yin / J. Xu \\ E-mail: yinjiajun_|@163.com / xujiaa_jj@163.com \\ Genet. Mol. Res. 14 (4): 16938-16947 (2015) \\ Received July 7, 2015 \\ Accepted September 29, 2015 \\ Published December 14, 2015 \\ DOI http://dx.doi.org/10.4238/2015.December.14.22
}

\begin{abstract}
The objective of this study was to investigate the mRNA expression of hepatic phosphoenolpyruvate carboxykinase (PEPCK) after gastric bypass surgery (GBS) in rats with type 2 diabetic mellitus (T2DM). Thirty-six male Goto-Kakizaki rats, aged 12 weeks, were randomly divided into the GBS, sham operation with diet restriction (SO), and sham operation alone (control) groups ( $\mathrm{N}=12$ per group). Liver specimens from all rats were obtained during the operation and 8 weeks after operation. Blood lipid levels were measured before and 8 weeks after operation. Fasting blood glucose (FBG), food intake, and body weight were recorded at weekly time points after operation. The blood glucose area under the curve (AUC) was calculated, and insulin sensitivity indices (ISI) were assessed. The expression PEPCK mRNA and protein were measured by real-time polymerase chain reaction and western blot. Compared with those of the SO and control groups, the blood lipid levels and the FBG in the GBS group was significantly decreased $(P<0.05)$, as was the AUC $(P<0.05)$, whereas the ISI was significantly increased $(P<0.05)$. PEPCK mRNA and protein levels in the GBS group were lower than those in the control
\end{abstract}


group, whereas those in the SO group were significantly higher than those in controls $(P<0.05)$. In conclusion, GBS can reduce blood glucose in T2DM rats while improving glucose tolerance and hyperglycemia, and the mechanism appears to be associated with a decrease of hepatic PEPCK mRNA and protein expression.

Key words: Gastric bypass surgery; Type 2 diabetes mellitus; Phosphoenolpyruvate carboxykinase; Gluconeogenesis

\section{INTRODUCTION}

Diabetes mellitus is a chronic disease that threatens human health, and $90 \%$ of affected individuals are diagnosed with type 2 diabetes mellitus (T2DM). Although T2DM is characterized by periods of high blood sugar, long-term control of blood glucose through dietary changes or by taking oral medications that reduce blood glucose levels is not ideal. Moreover, insulin replacement therapy only resolves the problem temporarily. Phosphoenolpyruvate carboxykinase (PEPCK) is one of the key rate-limiting enzymes in gluconeogenesis, and therefore the mRNA level of PEPCK is correlated with the rate of the gluconeogenesis process. In patients with T2DM, important physiological changes occur that include an increase in glycogen generation induced by insulin resistance, and an increase in gluconeogenesis, which is closely related to the overexpression of PEPCK (Cadoudal et al., 2008).

Recent research has shown that gastric bypass surgery (GBS) has a good therapeutic effect on T2DM. GBS not only improves blood glucose, insulin, and glycosylated hemoglobin levels, but it also rectifies impaired glucose tolerance and insulin resistance (Durbin, 2004). Furthermore, Mingrone et al. (2012) found that the therapeutic effect of GBS operation on T2DM was better than that of drug therapy alone. However, the molecular mechanisms underlying the effects of GBS remain unclear. Therefore, we examined changes in PEPCK mRNA and protein levels before and after GBS in Goto-Kakizaki (GK) rats, to gain insight into the influence of GBS on PEPCK expression, which would also provide evidence of the molecular mechanism of its hypoglycemic action.

\section{MATERIAL AND METHODS}

\section{Animals}

Thirty-six specific-pathogen-free (SPF) grade male GK rats, which develop T2DM early in life, were obtained from Vital River Laboratory Animal Technology Co., Ltd. (affiliate of Charles River Laboratories Inc., Beijing, China). Rats aged 12 weeks and weighing $335.3 \pm 10.8 \mathrm{~g}$ were fed in laboratory and randomly divided into the GBS group $(N=12)$, sham operation with diet restriction group (SO group) ( $\mathrm{N}=12)$, and sham operation alone group (control group) $(\mathrm{N}=12)$. The feedstuffs for a high-fat-diet (Research Diets Rodent Diet with $45 \% \mathrm{kcal} \%$ fat) were obtained from Shanghai Eu-Bio Co., Ltd. (Shanghai, China). Rats in the GBS group received GBS, rats in the SO group received diet restriction with the same food intake (FI) as the GBS group, and rats in the control group were given an unrestricted diet. All rats had unrestricted access to water.

\section{Surgical procedures}

Rats in the GBS group received gastric bypass surgery according to the following 
procedure. After 24-h fasting, the rats were anaesthetized with $10 \%$ chloral hydrate $(0.3 \mathrm{~g} / \mathrm{kg})$. The middle-lower side of the one-third lesser curvature and the higher-middle side of the onethird greater curvature were used as the boundary for partitioning the stomach with distal closure. The jejunum was partitioned $10 \mathrm{~cm}$ away from the Treitz ligament, and the distal jejunum was anastomosed with proximal gastrectomy. The proximal jejunum was anastomosed with the jejunum $10 \mathrm{~cm}$ away from the gastrointestinal anastomosis. Then the abdomen was flushed with $10 \mathrm{~mL}$ gentamicin $(80,000 \mathrm{U})$. After anesthesia recovery, rats were given free access to water, and were fed a high-fat diet after $48 \mathrm{~h}$.

The rats in the SO and control groups received exploratory laparotomy. Anastomosis in situ was carried out between the higher-middle side of the one-third lesser curvature and the proximal jejunum $20 \mathrm{~cm}$ away from the Treitz ligament, with $1 \mathrm{~cm}$ incisions and the same operation time as the GBS group.

Liver specimens $(200 \mathrm{mg}$ ) were taken from all rats during operation, which were then rinsed with normal saline, dried with sterile gauze, and stored in liquid nitrogen for later gene detection.

\section{Examination}

Total cholesterol (TC), triglyceride (TG), and free fatty acid (FFA) examination: Before and 8 weeks after operation, the TC and TG levels of all rats were measured by an Olympus AU400 Automatic Biochemical Analyzer (Olympus Optical Co., Ltd., Tokyo, Japan), and FFA was measured with an enzyme-linked immunosorbent assay (ELISA) kit (Lanpai Biotechnology Co., Ltd., Shanghai, China).

Fasting blood glucose (FBG), FI, and body weight (BW) examination: After operation, the FI and BW of all the rats were measured at 8 a.m. each day. FBG was measured with a OneTouch Ultra Easy Blood Glucose Meter (Johnson Medical Companies, Shanghai, China) before operation and each week thereafter. After 8 weeks, GK rats were euthanized and the liver tissues were harvested, rinsed with normal saline, dried with sterile gauze, and stored in liquid nitrogen for later gene detection.

Oral glucose tolerance test (OGTT): Before operation and 1, 4, and 8 weeks after operation, all rats received gastric perfusion with $50 \%$ glucose solution $(1.5 \mathrm{~g} / \mathrm{kg})$. Blood samples were collected from rat tails at different time points and blood glucose levels were measured at 0 , $0.5,1$, and $2 \mathrm{~h}$ after perfusion. The blood glucose area under the curve (AUC) was defined as: 0.25 $\mathrm{x}$ [(blood glucose at $0 \mathrm{~h})+4 \mathrm{x}($ blood glucose at $0.5 \mathrm{~h})+3 \mathrm{x}$ (blood glucose at $2 \mathrm{~h})]$, which was used to calculate the AUC of "blood glucose - concentration".

Insulin sensitivity indices (ISI): Before operation and 1, 4, and 8 weeks after operation, the fasting serum insulin (FSI) of all rats was measured at each time point, and ISI were calculated using the following formulae: ISI $1=1 /(\mathrm{FBG} \times \mathrm{FSI})$ and ISI $=\ln (\mathrm{FBG} \times \mathrm{FSI})-1$.

\section{Measurement of PEPCK mRNA levels by RT-PCR}

Liver tissues (100 mg; those stored in liquid nitrogen) were cut into small particles with scissors, and ground to fine powder with a mortar and pestle in liquid nitrogen. Then $1 \mathrm{~mL}$ TRIzol Reagent (Thermo Fisher Scientific Inc., Shanghai, China) was added and the total RNA was extracted according to manufacturer specifications. Subsequently, $1 \mu \mathrm{L}$ total RNA was diluted with diethylpyrocarbonate (DEPC) water $(1: 100)$ and the concentration and purity of the RNA measured with the NanoDrop 2000 ultraviolet spectrophotometer (Thermo Fisher Scientific Inc.), with an optical density (OD) $A_{260} / A_{280}$ ratio between 1.8 and 2.0. According to sample concentration, the 
RNA was diluted with DEPC water to a terminal concentration of $1 \mathrm{mg} / \mathrm{mL}$. PEPCK and $\beta$-actin (internal control) PCR primers were synthesized by Shanghai Sangon Biotech Co., Ltd. (Table 1). Subsequently, $25 \mu \mathrm{L}$ SuperScript ${ }^{\circledR}$ One-Step RT-PCR reactions were prepared (Table 2) according to the manufacturer instructions with Platinum ${ }^{\circledR}$ Taq DNA Polymerase (Thermo Fisher Scientific Inc.). PCR amplification was then performed with a BIO-RAD C1000, and the PEPCK mRNA levels were quantified using the $2^{-\Delta \Delta \mathrm{Ct}}$ method where $\Delta \Delta \mathrm{Ct}=\Delta \mathrm{Ct}$ (sample) $-\Delta \mathrm{Ct}$ (standard) normalize to $\beta$-actin (Table 3).

\begin{tabular}{lllc}
\multicolumn{2}{c}{ Table 1. Primers for RT-PCR amplification. } \\
\hline Gene & & Primer sequences & Amplicon length (bp) \\
\hline PEPCK & Forward & 5'-GCCTGGGGGACGTCAACGTG-3' & 247 \\
& Reverse & 5'-CCTAGCTCCCAACGCGCGTGCC-3' & 619 \\
& Forward & 5'-ACGGTCAGGTCATCACTATCG-3' & \\
\hline
\end{tabular}

\begin{tabular}{lc}
\multicolumn{1}{c}{ Table 2. RT-PCR set up. } \\
\hline Component & Volume $(\mu \mathrm{L})$ \\
\hline Ultrapure water & 8.75 \\
2X PCR Mix & 12.5 \\
Primer F & 1.25 \\
$\quad$ R & 1.25 \\
Template DNA & 1.25 \\
Total volume & 25.0 \\
\hline
\end{tabular}

\begin{tabular}{|c|c|c|c|}
\hline Step & Temperature $\left({ }^{\circ} \mathrm{C}\right)$ & Time & Cycles \\
\hline Predegeneration & 94 & $5 \mathrm{~min}$ & 30 cycles \\
\hline Degeneration & 94 & $30 \mathrm{~s}$ & \\
\hline Annealing & $59-61$ & $30 \mathrm{~s}$ & \\
\hline Extension & 72 & $30 \mathrm{~s}$ & \\
\hline Extension terminal & 72 & $7 \mathrm{~min}$ & 1 cycle \\
\hline Storage & 4 & $\rightarrow \infty$ & \\
\hline
\end{tabular}

\section{Measurement of PEPCK protein levels}

Proteins from liver tissues were extracted with a protein extraction kit (Nanjing KeyGEN BioTECH Development Co., Ltd., Nanjing, China) and stored at $-80^{\circ} \mathrm{C}$. Protein concentration was determined by the bicinchoninic acid (BCA) assay (Beijing Solarbio Technology Co., Ltd., Beijing, China). Then, $40 \mu \mathrm{g}$ protein samples were analyzed by sodium dodecyl sulfate-polyacrylamide gel electrophoresis (SDS-PAGE) with a $5 \%$ stacking gel and $10 \%$ separating gel and transferred to a polyvinylidene fluoride (PVDF) membrane (Millipore Corporate, Billerica, MA, USA). Following transfer, membranes were blocked with Tris-buffered saline (TBS) (Wuhan Boster Bio-engineering Co., Ltd., Wuhan, China) containing $0.05 \%$ Tween-20 and 5\% non-fat milk for $2 \mathrm{~h}$ at room temperature, then incubated with an anti-PEPCK antibody and an anti- $\beta$-actin antibody (Santa Cruz Biotechnology, Inc., Santa Cruz, CA, USA) each diluted 1:1000 at $4^{\circ} \mathrm{C}$ overnight, and finally incubated with an appropriate goat anti-rabbit antibody and goat anti-mouse antibody (Jackson, 
Shanghai Haoran Biological Technology Co., Ltd., Shanghai, China) each diluted 1:3000 for $1 \mathrm{~h}$ at room temperature. After washing with TBST (TBS + 0.01\% Tween), membrane exposure was carried out with the ECL Plus chemiluminescent kit (Beijing Solarbio Technology Co., Ltd., Beijing, China), and the bands were visualized and quantified with the UVP BioSpectrum 410 Imaging System.

\section{Statistical analysis}

All data were analyzed using SPSS 17.0 (SPSS Inc., Chicago, IL, USA). Measurement data are reported as means \pm standard deviation. Comparisons between groups were performed by one-way ANOVA. Differences in indices between groups were analyzed by Fisher least significant difference (LSD) tests. Comparisons of data before and after operations were performed by paired $t$-tests. $\mathrm{P}$ values $<0.05$ were considered to be significant.

\section{RESULTS}

\section{Blood-lipoid levels for each group}

After 8 weeks, the TC, TG, and FFA levels of rats in the GBS group were significantly lower compared to those in the SO and control groups $(P<0.05)$ (Table 4).

Table 4. Blood-lipoid levels for each group (means $\pm \mathrm{SD}$, all values shown in $\mathrm{mM}, \mathrm{N}=12$ per group).

\begin{tabular}{lccccccc}
\hline Groups & \multicolumn{3}{c}{ Pre-operation } & & \multicolumn{3}{c}{8 weeks post-operation } \\
\cline { 2 - 4 } & TC & TG & FFA & & TC & TG & FFA \\
\hline GBS & $2.31 \pm 0.52$ & $1.44 \pm 0.27$ & $1.08 \pm 0.06$ & & $1.25 \pm 0.08^{\text {*\# }}$ & $0.93 \pm 0.10^{\text {*\# }}$ & $0.88 \pm 0.12^{\text {*\# }}$ \\
SO & $2.40 \pm 0.39$ & $1.49 \pm 0.20$ & $1.10 \pm 0.04$ & & $2.37 \pm 0.19$ & $1.37 \pm 0.22$ & $1.14 \pm 0.10$ \\
Control & $2.37 \pm 0.47$ & $1.39 \pm 0.25$ & $1.01 \pm 0.04$ & & $2.41 \pm 0.20$ & $1.50 \pm 0.26$ & $1.17 \pm 0.13$ \\
\hline
\end{tabular}

GBS = gastric bypass surgery; $\mathrm{SO}=$ shaw operation. ${ }^{*} \mathrm{P}<0.05$ compared with control group; ${ }^{\#} \mathrm{P}<0.05$ compared with SO group.

\section{FBG, FI and FFA changes}

Before operation, there were no significant differences in the FBG among the three groups $(P=0.981)$. After operation, the FBG in the GBS group was $17.44 \pm 1.12 \mathrm{mM}$, and decreased at subsequent time points significantly more than that in the $S O$ group and control group $(P<0.05)$. From 1 to 8 weeks after operation, the FBG in the GBS group was lower compared with that in the SO group and control group, while the FBG in the SO group was significantly lower compared with that in the control group $(P<0.05)$ (Figure 1).

Before operation, there were no significant differences in the $\mathrm{FI}$ among the three groups $(P=0.65, P=0.41)$. After operation, the $F I$ in the GBS group decreased in the 1st and 2nd week compared with that pre-operation, and remained at the reduced level from the 3rd to the 8th week (140 g/week). Compared with that in the control group, the FI in the GBS group was significantly decreased $(P<0.05)$ (Figure 2).

Within the 1 st week after operation, the BW of rats in the GBS group significantly decreased from that of pre-operation $(349.83 \pm 16.62 \mathrm{~g})$ to that of post-operation $(293.56 \pm 20.06 \mathrm{~g})(\mathrm{P}<0.05)$. After 6 weeks, the BW of the GBS group had increased to that of the pre-operative level (338.45 \pm $16.29 \mathrm{~g}$ ), which was relatively later than that of the SO and control groups (Figure 3). 


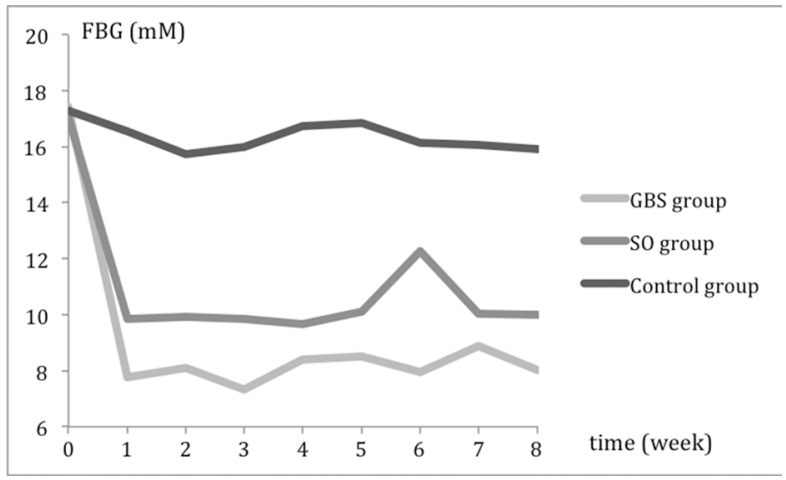

Figure 1. Changes in fasting blood glucose (FBG) before and after operation in GBS, SO, and control groups.

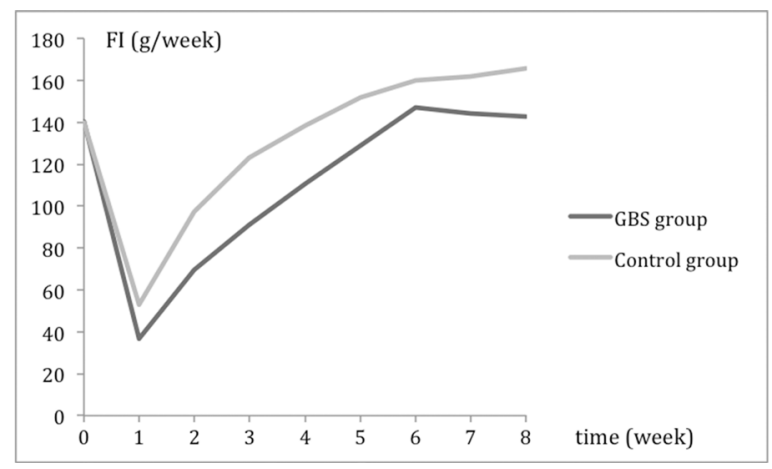

Figure 2. Changes in food intake (FI) before and after operation in GBS and control groups.

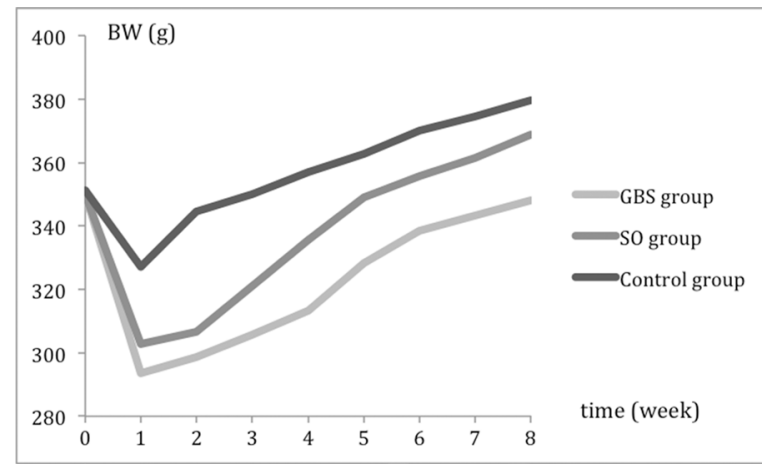

Figure 3. Changes in body weight (BW) before and after operation in GBS, SO, and control groups.

\section{Blood glucose}

The FBG and OGTT at $2 \mathrm{~h}$ after operation were decreased in the GBS group compared to those of the SO and control groups. Eight weeks later, the FBG and OGTT in the GBS group were significantly lower than those in the SO group control groups $(P<0.05)$ (Table 5$)$. 
Table 5. Blood glucose levels in each group (means \pm SD, all values shown in $\mathrm{mM}, \mathrm{N}=12$ per group).

\begin{tabular}{|c|c|c|c|c|c|c|c|c|}
\hline \multirow[t]{2}{*}{ Groups } & \multicolumn{2}{|c|}{ Pre-operation } & \multicolumn{2}{|c|}{1 week post-operation } & \multicolumn{2}{|c|}{4 weeks post-operation } & \multicolumn{2}{|c|}{8 weeks post-operation } \\
\hline & $\mathrm{Oh}$ & $2 \mathrm{~h}$ & $0 \mathrm{~h}$ & $2 \mathrm{~h}$ & $0 \mathrm{~h}$ & $2 \mathrm{~h}$ & $\mathrm{Oh}$ & $2 \mathrm{~h}$ \\
\hline GBS & $12.54 \pm 1.21$ & $17.73 \pm 1.49$ & $7.80 \pm 0.71^{\star \#}$ & $8.83 \pm 0.75^{\star \#}$ & $6.70 \pm 0.39^{* \#}$ & $8.28 \pm 0.76^{\star \#}$ & $6.23 \pm 0.42^{\star \#}$ & $7.98 \pm 0.63^{* \#}$ \\
\hline so & $11.99 \pm 1.30$ & $17.80 \pm 1.50$ & $12.59 \pm 1.48$ & $17.89 \pm 1.30$ & $12.99 \pm 1.35$ & $20.40 \pm 1.79$ & $13.58 \pm 0.97$ & $24.01 \pm 1.88$ \\
\hline Control & $12.38 \pm 1.27$ & $18.01 \pm 1.45$ & $12.88 \pm 1.39$ & $18.19 \pm 1.44$ & $13.02 \pm 1.27$ & $20.24 \pm 1.49$ & $13.25 \pm 1.04$ & $23.05 \pm 1.98$ \\
\hline
\end{tabular}

${ }^{*} \mathrm{P}<0.05$ compared with control group; ${ }^{*} \mathrm{P}<0.05$ compared with SO group.

AUC

Compared with that in the SO and control groups, the AUC in the GBS group was significantly decreased $(P<0.05)$ (Table 6).

Table 6. AUC for each group (means $\pm \mathrm{SD}$, all values shown in $\mathrm{mM}, \mathrm{N}=12$ per group).

\begin{tabular}{lcccc}
\hline Groups & Pre-operation & 1 week post-operation & 4 weeks post-operation & 8 weeks post-operation \\
\hline GBS & $38.94 \pm 1.99$ & $25.38 \pm 1.67^{* \#}$ & $22.03 \pm 1.40^{* \#}$ & $19.89 \pm 1.58^{* \#}$ \\
SO & $38.78 \pm 2.05$ & $39.41 \pm 2.30$ & $42.59 \pm 2.76$ & $43.27 \pm 2.10$ \\
Control & $39.02 \pm 2.21$ & $40.20 \pm 2.52$ & $44.02 \pm 2.73$ & $45.90 \pm 2.35$ \\
\hline
\end{tabular}

${ }^{*} \mathrm{P}<0.05$ compared with control group; ${ }^{\mathrm{P}} \mathrm{P}<0.05$ compared with $\mathrm{SO}$ group.

\section{Insulin (mIU/L) and ISI}

One week after operation, the ISI in the GBS group were significantly increased compared with those in the SO and control groups, while the insulin level was significantly decreased $(P<$ 0.05). The effect of GBS was sustained through the 8th week (Table 7).

Table 7. Insulin (mIU/L) and ISI for each group (means \pm SD, $N=12$ per group).

\begin{tabular}{|c|c|c|c|c|c|c|c|c|}
\hline \multirow[t]{2}{*}{ Groups } & \multicolumn{2}{|c|}{ Pre-operation } & \multicolumn{2}{|c|}{1 week post-operation } & \multicolumn{2}{|c|}{4 weeks post-operation } & \multicolumn{2}{|c|}{8 weeks post-operation } \\
\hline & Insulin & ISI & Insulin & ISI & Insulin & ISI & Insulin & ISI \\
\hline GBS & $32.30 \pm 3.79$ & $-5.78 \pm 0.10$ & $17.89 \pm 3.30^{\text {*\# }}$ & $-4.86 \pm 0.14^{\star \#}$ & $17.67 \pm 2.13^{\text {*\# }}$ & $-4.72 \pm 0.13^{\text {*\# }}$ & $20.55 \pm 3.32^{\star \#}$ & $-4.64 \pm 0.15^{\text {*\# }}$ \\
\hline so & $31.44 \pm 3.52$ & $-6.03 \pm 0.09$ & $28.87 \pm 2.32$ & $-5.76 \pm 0.19$ & $26.91 \pm 2.25$ & $-5.85 \pm 0.14$ & $29.32 \pm 4.27$ & $-5.99 \pm 0.15$ \\
\hline Control & $31.46 \pm 3.88$ & $-5.84 \pm 0.08$ & $28.05 \pm 2.80$ & $-5.85 \pm 0.12$ & $27.07 \pm 3.13$ & $-5.70 \pm 0.11$ & $32.04 \pm 4.96$ & $-5.96 \pm 0.20$ \\
\hline
\end{tabular}

${ }^{*} \mathrm{P}<0.05$ compared with control group; $\mathrm{P}<0.05$ compared with $\mathrm{SO}$ group.

\section{PEPCK mRNA expression in liver tissues of rats}

Eight weeks after operation, the expression of PEPCK mRNA (relative to $\beta$-actin) was significantly lower in the GBS group $(3.97 \pm 0.30)$ than that in the SO group $(6.35 \pm 0.44)$ and control group $(6.09 \pm 0.72)(P<0.05)$.

\section{PEPCK protein expression in liver tissues of rats}

Eight weeks after operation, the expression of PEPCK protein (relative to $\beta$-actin) was significantly lower in the GBS group $(1.60 \pm 0.31)$ than that in the SO group $(1.98 \pm 0.24)$ and control group $(1.94 \pm 0.38)(P<0.05)$ (Figure 4). 

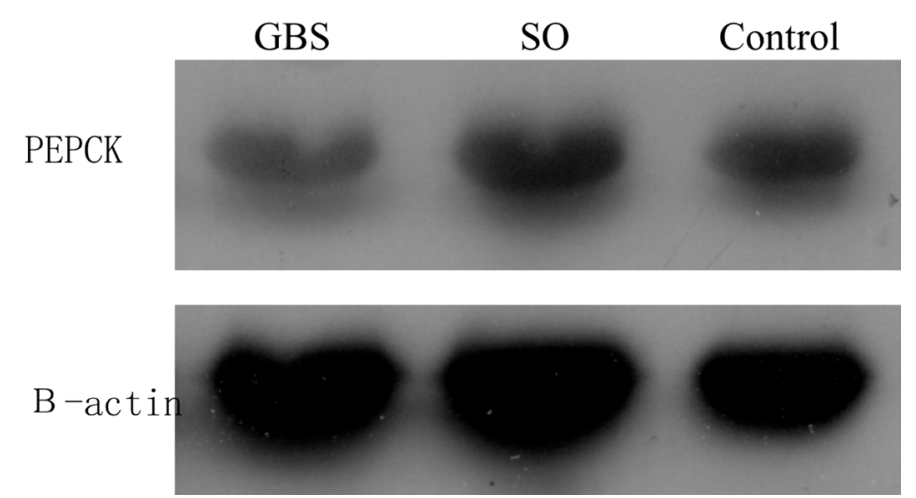

Figure 4. Western blot showing expression of the PEPCK protein in the livers of each group.

\section{DISCUSSION}

Many prior studies have demonstrated that GBS has a great effect on the treatment of diabetes mellitus. After GBS, the FBG, insulin, and glycosylated hemoglobin in $84-98 \%$ of patients with T2DM recovered to normal levels (Buchwald et al., 2009). Additionally, GBS improved the blood lipid profile, blood pressure, and insulin resistance in T2DM patients, and also reduced the risk and incidence of diabetic complications (Rubino et al., 2010). Despite this, the mechanism of improving glucose tolerance following GBS has remained unclear. The renal system plays an important role in maintaining the stability of blood glucose. The marked increases of glycogenolysis and gluconeogenesis are the main reasons for the increase of FBG in patients with T2DM (Cadoudal et al., 2008; D’Alessio, 2011).

Due to insulin resistance, T2DM decreases the sensitivity of organs and tissues to insulin, which leads to decreased utilization of glucose whereas it increases the generation of endothelial glucose. Two sources of endothelial glucose include the decomposition of glycogen and gluconeogenesis. Both these mechanisms are enhanced in patients with T2DM, which leads to hyperglycemia before and after meals.

Compared to healthy rats, GK rats, which were the experimental T2DM model herein, exhibit higher blood lipid and glucose levels, while the ISI are lower and glucose tolerance is damaged. One week after GBS, the FBG in GK rats was significantly decreased, insulin sensitivity was increased, and the damaged glucose tolerance was improved, which indicates that the hypoglycemic effect of GBS was strong and rapid. Eight weeks after GBS, blood glucose levels tended to be stable. The mRNA and protein expression levels of PEPCK, a gluconeogenic enzyme, were decreased compared to those in the control group, which indicates that gluconeogenesis was weakened. The decrease in gluconeogenesis may be related to the increase in insulin sensitivity. For instance, it has been reported that insulin can negatively regulate gluconeogenesis through the downregulation of gluconeogenic enzyme expression (Houde et al., 2010; Ip et al., 2012; DeFronzo et al., 2012), and can inhibit the generation of endothelial glucose in the liver and kidney (Pillot et al., 2009). Therefore, we predicted that the decrease of glycogen output might be related to the effect of insulin. After GBS, the secretion of many gastrointestinal hormones and adipocyte factors also changes (Swarbrick et al., 2008; de Hollanda et al., 2014). Whether there are other elements influenced by GBS still needs further investigation.

In the current study, GBS had a strong glucose-lowering effect in rats with T2DM. Two 
weeks after operation, there was no significant difference in the BW between the GBS group and the SO group $(P>0.05)$. However, there was a pronounced glucose-lowering effect observed in the GBS group, which suggests that there was an independent glucose-lowering mechanism from aside from the decrease in BW associated with GBS, and this is in agreement with previous results (Laferrere, 2011). Compared to that in the pre-operation and control groups, the PEPCK mRNA expression in liver tissues was significantly decreased after GBS $(P<0.05)$, which we predict may be the aforementioned independent mechanism leading to its glucose-lowering effect. Therefore, the decrease in PEPCK mRNA may be an important physiological change, which may be related to the improvements in the conditions of patients with T2DM after GBS, especially in decreasing FBG.

It was previously shown that after the complete gene knockout of PEPCK, rats died from hypoglycemia within 3 days of birth, whereas blood glucose could maintain at normal levels in rats with a partial gene knockout of PEPCK (She et al., 2003). Conversely, there is an increase in the output of glycogen and development of T2DM in rats with over expression of PEPCK (Valera et al., 1994). A recent study showed that the expression of cytosolic PEPCK (PCPCK), the key enzyme in gluconeogenesis in hepatocytes, was increased in mice with diabetes mellitus (Foretz et al., 2010). It was further shown that PCPCK plays an important role in maintaining blood glucose and insulin resistance.

Many hypoglycemic drugs, such as metformin, work by effectively inhibiting gluconeogenesis, which decreases the expression of PCPCK and restores blood glucose levels and ISI. Prior research has demonstrated that physical exercise and traditional Chinese medicine extracts used to treat diabetes had a similar hypoglycemic effect (Chang et al., 2006; Choi et al., 2008). Currently, there are many medications for treating diabetes mellitus that successfully inhibit gluconeogenesis. However, whether GBS worked through a similar mechanism remained unclear. In the current study, we found that GBS reduced PEPCK mRNA expression in the livers of GK rats with T2DM, indicating that GBS inhibits the expression of PEPCK mRNA, which in turn inhibits gluconeogenesis and reduces blood glucose. Moreover, changes in gastrointestinal hormones including ghrelin, glucose-dependent insulinotropic polypeptide (GIP), glucagon-like peptide-1 (GLP-1), and peptide-tyrosine-tyrosine (PYY) may improve the activity of insulin and the uptake and utilization of glucose in the entire body, while also inhibiting glycogenolysis and gluconeogenesis, and reduce the generation of endogenous blood glucose to reduce total blood glucose. However, the concrete mechanisms underlying the effects of GBS on T2DM are still unclear.

In the present study, the FBG of rats in the SO group was significantly decreased compared to that in the pre-operation and control group, while there was no change in PEPCK mRNA expression. In healthy human and animals, the liver maintains the stability of blood glucose levels by enhancing glycogenolysis and gluconeogenesis when the body is under fasting conditions (Staehr et al., 2004). Our results indicate that when GK rats were under dietary restriction, a similar physiological response to that of healthy rats was induced by enhancing gluconeogenesis to maintain blood glucose levels. This phenomenon indicates that dietary restriction can improve blood glucose in rats with diabetes mellitus at the molecular level, but may not be successful for treating diabetes mellitus alone. Taken together, the results herein indicate that the blood glucose reduction after GBS in patients with T2DM is related to the decrease in PEPCK mRNA expression.

\section{Conflicts of interest}

The authors declare no conflict of interest. 


\title{
ACKNOWLEDGMENTS
}

\author{
Research supported by the Natural Science Foundation of Liaoning Province (\#201102006).
}

\section{REFERENCES}

Buchwald H, Estok R, Fahrbach K, Banel D, et al. (2009). Weight and type 2 diabetes after bariatric surgery: systematic review and meta-analysis. Am. J. Med. 122: 248-256.

Cadoudal T, Fouque F, Benelli C and Forest C (2008). Glyceroneogenesis and PEPCK-C: pharmacological targets in type 2 diabetes. Med. Sci. (Paris) 24: 407-413.

Chang SP, Chen YH, Chang WC, Liu IM, et al. (2006). Merit of physical exercise to reverse the higher gene expression of hepatic phosphoenolpyruvate carboxykinase in obese Zucker rats. Life Sci 13: 240-246.

Choi MS, Jung UJ, Yeo J, Kim MJ, et al. (2008). Genistein and daidzein prevent diabetes onset by elevating insulin level and altering hepatic gluconeogenic and lipogenic enzyme activities in non-obese diabetic (NOD) mice. Diabetes Metab. Res. Rev. 24: 74-81.

D'Alessio D (2011). The role of dysregulated glucagon secretion in type 2 diabetes. Diabetes Obes. Metab. 1: 126-132.

de Hollanda A, Jimenez A, Corcelles R, Lacy AM, et al. (2014). Gastrointestinal hormones and weight loss response after Roux-en-Y gastric bypass. Surg. Obes. Relat. Dis.10: 814-819.

DeFronzo RA, Davidson JA and Del Prato S (2012). The role of the kidneys in glucose homeostasis: a new path towards normalizing glycaemia. Diabetes Obes. Metab. 14: 5-14.

Durbin RJ (2004). Thiazolidinedione therapy in the prevention/delay of type 2 diabetes in patients with impaired glucose tolerance and insulin resistance. Diabetes Obes. Metab. 6: 280-285.

Foretz M, Hebrard S, Leclerc J, Zarrinpashneh E, et al. (2010). Metformin inhibits hepatic gluconeogenesis in mice independently of the LKB1/AMPK pathway via a decrease in hepatic energy state. J. Clin. Invest. 120: 2355-2369.

Houde VP, Brule S, Festuccia WT, Blanchard PG, et al. (2010). Chronic rapamycin treatment causes glucose intolerance and hyperlipidemia by upregulating hepatic gluconeogenesis and impairing lipid deposition in adipose tissue. Diabetes 59: 1338-1348.

Ip W, Shao W, Chiang YT and Jin T (2012). The Wnt signaling pathway effector TCF7L2 is upregulated by insulin and represses hepatic gluconeogenesis. Am. J. Physiol. Endocrinol. Metab. 303: E1166-E1176.

Laferrere B (2011). Do we really know why diabetes remits after gastric bypass surgery? Endocrine 40: 162-167.

Mingrone G, Panunzi S, De Gaetano A, Guidone C, et al. (2012). Bariatric surgery versus conventional medical therapy for type 2 diabetes. N. Engl. J. Med. 366: 1577-1585.

Pillot B, Soty M, Gautier-Stein A, Zitoun C, et al. (2009). Protein feeding promotes redistribution of endogenous glucose production to the kidney and potentiates its suppression by insulin. Endocrinology 150: 616-624.

Rubino F, Schauer PR, Kaplan LM, Cummings DE (2010). Metabolic surgery to treat type 2 diabetes: clinical outcomes and mechanisms of action. Annu. Rev. Med. 61: 393-411.

She P, Burgess SC, Shiota M, Flakoll P, et al. (2003). Mechanisms by which liver-specific PEPCK knockout mice preserve euglycemia during starvation. Diabetes 52: 1649-1654.

Staehr P, Hother-Nielsen $\mathrm{O}$ and Beck-Nielsen H (2004). The role of the liver in type 2 diabetes. Rev. Endocr. Metab. Disord. 5: $105-110$.

Swarbrick MM, Stanhope KL, Austrheim-Smith IT, Van Loan MD, et al. (2008). Longitudinal changes in pancreatic and adipocyte hormones following Roux-en-Y gastric bypass surgery. Diabetologia 51: 1901-1911.

Valera A, Pujol A, Pelegrin M and Bosch F (1994). Transgenic mice overexpressing phosphoenolpyruvate carboxykinase develop non-insulin-dependent diabetes mellitus. Proc. Natl. Acad. Sci. U.S.A. 91: 9151-9154. 